Chirurg 2021 · 92:918-923

https://doi.org/10.1007/s00104-021-01463-0

Angenommen: 22. Juni 2021

Online publiziert: 23. Juli 2021

๑) Springer Medizin Verlag GmbH, ein Teil von

Springer Nature 2021

Redaktion

C. Bruns, Köln

\section{D}

\title{
Auswirkung der COVID-19- Pandemie auf die hepatopankreatobiliäre Chirurgie und die Organtransplantation
}

\author{
K. Hillebrandt · N. Nevermann · B. Globke · S. Moosburner · M. Schmelzle · \\ Johann Pratschke \\ Chirurgische Klinik, Campus Charité Mitte | Campus Virchow-Klinikum, Charité - Universitätsmedizin \\ Berlin, Berlin, Deutschland
}

\section{Zusammenfassung}

\section{In diesem Beitrag}

- Auswirkung der COVID-19-Pandemie auf die HPB-Programme

- Auswirkung der COVID-19-Pandemie auf die Transplantationsprogramme

- Risikokonstellation perioperative SARSCoV-2-Infektion

- Risikokonstellation Organtransplantation

- Ausblick: Impfung

Die Autoren K. Hillebrandt, N. Nevermann und B. Globke teilen sich die Erstautorenschaft.

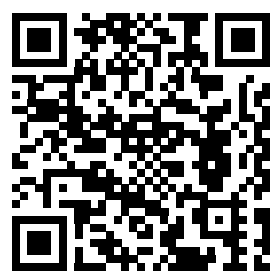

QR-Code scannen \& Beitrag online lesen

Hintergrund und Ziel der Arbeit: Im Rahmen der COVID-19("coronavirus disease 2019")-Pandemie wurden weitreichende Ressourceneinschränkungen und -umverteilungen innerhalb des Gesundheitssystems notwendig. Diese Übersichtsarbeit beleuchtet die direkten und indirekten Folgen der COVID-19Pandemie auf die Bereiche der hepatopankreatobiliären (HPB)-Chirurgie und Organtransplantation unter Berücksichtigung aktueller Literatur und veröffentlichter Expertenmeinungen nationaler und internationaler Fachgesellschaften. Entwicklungen der Operationszahlen wurden über die Eurotransplant Statistics Report Library und eine zentrumsinterne Auswertung HPB-chirurgischer Eingriffe analysiert.

Ergebnisse: In den Bereichen der HPB-Chirurgie und Organtransplantation zeigte sich vor allem in der ersten Pandemiewelle an vielen Zentren ein deutlicher Einbruch der Eingriffszahlen. Interessanterweise konnte meist kein kompensatorischer Zuwachs der Operationen in den Folgemonaten verzeichnet werden. Im Jahrestrend zeigen sich somit leicht rückläufige Zahlen. Ob dieser Trend auf eine erhöhte Sterblichkeit durch verschobene Operationen und geänderte Therapieregimes zurückzuführen ist, lässt sich derzeit zumindest nicht ausschließen. Eine SARS-CoV-2(,severe acute respiratory syndrome coronavirus $2^{\prime \prime}$ )-Infektion ist auf jeden Fall mit einem komplikativen Verlauf assoziiert, insbesondere im perioperativen Verlauf und nach Transplantation.

Diskussion: Aufgrund des erhöhten Risikoprofils der genannten Patientengruppen besteht ein erhöhtes Risiko für einen schweren COVID-19-Verlauf. Dies muss bei der Abwägung von Therapiealternativen, der Schutzempfehlungen und der Priorisierung bei Impfungen in Betracht gezogen werden.

\section{Schlüsselwörter}

SARS-CoV-2 · Perioperative Infektion · Versorgungssituation · Impfung · Immunsuppression

Die COVID-19-Pandemie führte zu einer bislang nicht gekannten Notwendigkeit an Ressourceneinschränkungen und -umverteilungen mit mannigfaltigen Auswirkungen auf alle Bereiche des Gesundheitssystems. Aufgrund weit überdurchschnittlich hoher struktureller und personeller Vorhaltungen, die für die Aufrechterhaltung hepatopankreatobiliärer (HPB)- und transplantationschirurgischer Programme notwendig sind, ist die Patientenversorgung in die- sen Bereichen von pandemieassoziierten Strukturveränderungen besonders stark betroffen.

Auswirkung der COVID-19Pandemie auf die HPB-Programme

$\mathrm{Zu}$ Beginn der Pandemie wurde von nationalen und internationalen chirurgischen Fachgesellschaften eine Vielzahl orientierender Handlungsempfehlungen veröffentlicht, in welcher Form der Reduk- 


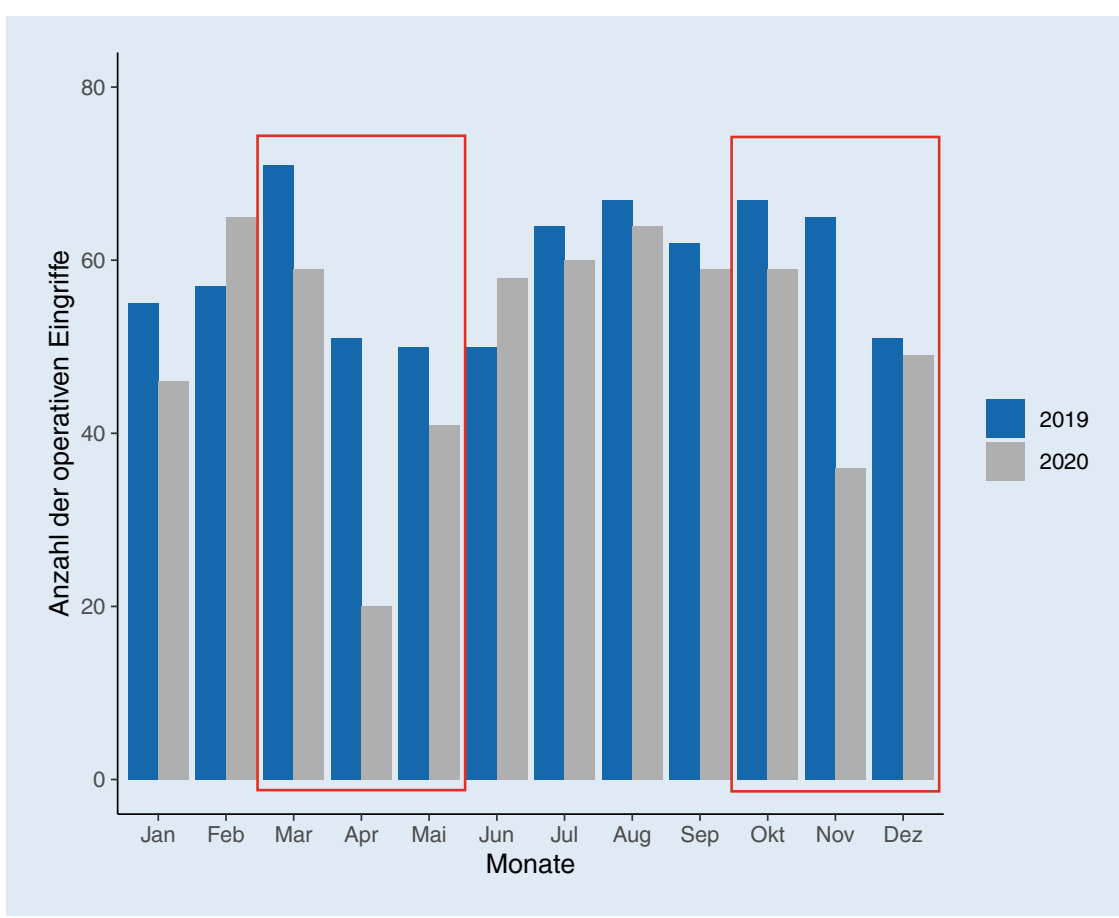

Abb. $1 \Delta$ Anzahl der hepatopankreatobiliärer Eingriffe an der Chirurgischen Klinik der Charité (Campus Charité Mitte und Campus Virchow-Klinikum) im Jahresverlauf (2019vs. 2020). Die rot eingerahmten Kästen markieren die Pandemiehochphasen in Deutschland

tion von Operationskapazitäten inhaltlich durch eine Stratifizierung sinnvollerweise begegnet werden kann. Entsprechend der hohen therapeutischen Dringlichkeit sollten notfallmäßige, onkologische Eingriffe und lebenswichtige Transplantationen priorisiert werden. In einer von unserer Klinik initiierten europaweiten Onlineumfrage im April 2020 zeigte sich allerdings, dass es in der ersten Welle in ca. $50 \%$ der HPB-Programme zu Einschränkungen kam, mit einem von den Teilnehmern erwarteten negativen Einfluss auf das (onkologische) Outcome der Patienten [23]. Als Ursache hierfür wurden eine reduzierte Verfügbarkeit von Intensivbetten angegeben sowie eine notwendige Reduktion der Operationskapazitäten. Sogar eine konsekutive Notwendigkeit der Triagierung von Patienten wurde berichtet. Interessanterweise wurde auch eine Reduktion in der Patientenzuweisung für europäische HPBZentren beschrieben [23]. Diese Entwicklungen waren im Großraum Paris z.B. mit einer signifikanten Reduktion der Neudiagnosen eines hepatozellulären Karzinoms (HCC) und verlängerten Intervallen bis zum Therapiebeginn assoziiert [4].
Das American College of Surgeon (ACS) empfahl die Verwendung der Elective Surgery Acuity Scale (ESAS) für eine entsprechende Kategorisierung chirurgischer Eingriffe. Onkologische Eingriffe, die den Großteil der HPB-Chirurgie ausmachen, wurden hierbei als Tier $3 \mathrm{~b}$ "do not postpone" klassifiziert. Des Weiteren wurde die Frage nach einer Modifizierung von Therapiekonzepten hin zu ressourcensparenden Optionen diskutiert. Bennett et al. bieten eine umfassende Übersicht und Empfehlung bezüglich überbrückender Therapiekonzepte, falls eine Operation pandemiebedingt nicht möglich sein sollte. Dabei handelt es sich um interventionelle und sonstige (vermeintlich) ressourcensparenden Alternativen zu einer chirurgischen Intervention, z.B. Chemoablation statt Resektion beim HCC oder systemische Therapie beim Kolorektalkarzinom [6]. Es ist festzuhalten, dass die Alternativen mit ungleich schlechteren Therapieergebnissen verbunden sind und nur als temporäre Ultima ratio für hochgradig überlastete Gesundheitssysteme zu klassifizieren sind.

Die Zahlen unserer Klinik zeigen einen deutlichen Einbruch während der ersten
Welle. Im weiteren Jahresverlauf zeigte sich zwar eine Stabilisierung, jedoch ohne den erwarteten kompensatorischen Anstieg der Operationszahlen ( $\bullet$ Abb. 1). Dass HPB-Chirurgie auch während einer Pandemie sinnvoll weitergeführt werden kann, zeigt eine Zentrumserfahrung aus Großbritannien, die sowohl stabile Operationszahlen als auch ein vergleichbares Outcome zum Vorjahreszeitraum beschreibt [3].

\section{Auswirkung der COVID-19- Pandemie auf die Trans- plantationsprogramme}

Die Organtransplantation war insbesondere in der ersten Welle schwer durch die Pandemie betroffen mit einem international sichtbaren, drastischen Rückgang der Transplantationsaktivität. Zwischen Februar und März verzeichnete Frankreich einen Rekordrückgang der durchgeführten Transplantationen um $90 \%$ [19]. Für die Niederlande wurde ein Rückgang um über $60 \%$ innerhalb des ersten Monats der Pandemie verzeichnet [28]. Einerseits war eine bewusste Schonung der Kapazitäten des Gesundheitssystems (v. a. Intensivbetten) Grund für die Reduktion der Aktivität, andererseits verursachte die pandemische Ausbreitung von SARS-CoV-2 eine Verzerrung der Nutzen-Risiko-Bewertung. Dies wurde insbesondere deutlich bei vermeintlich aufschiebbaren Transplantationen nach Nierenlebendspende. Im internationalen Konsens wurde empfohlen, Lebendspendeprogramme und elektive Transplantationen zu reduzieren und gleichzeitig eine fortlaufende Versorgung akut lebensrettender Transplantationen zu sichern [27].

\section{\) Der Rückgang bei der Nierentransplantation nach Lebendspende betrug $21 \%$}

Eurotransplant (ET) hat im Januar 2021 die Jahresstatistiken für 2020 veröffentlicht: Über das gesamte Jahr 2020 hinweg zeigt sich ein nur leichter Rückgang der Organtransplantationen um 9\% im Vergleich zu 2019 (2019: 6981 Organe von 2042 Spendern; 2020: 6356 Organe von 1837 Spendern; [29]). Den Empfehlungen mehrerer Fachgesellschaften folgend war der Rückgang bei der Nierentransplantati- 


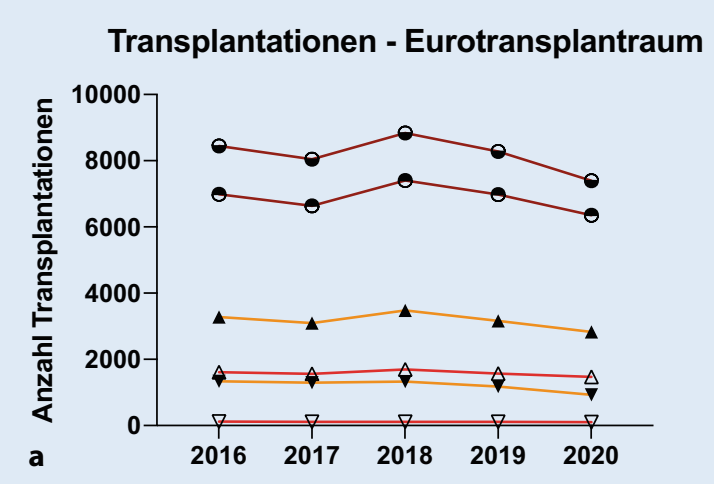

- Alle Transplantationen, Totenspende

- Alle Transplantationen, Lebendspende

$\nabla$ Lebertransplantation, Lebendspende

$\triangle$ Lebertransplantation, Totenspende

、 Nierentransplantation, Lebendspende

- Nierentransplantation, Totenspende

Transplantationen - Deutschland

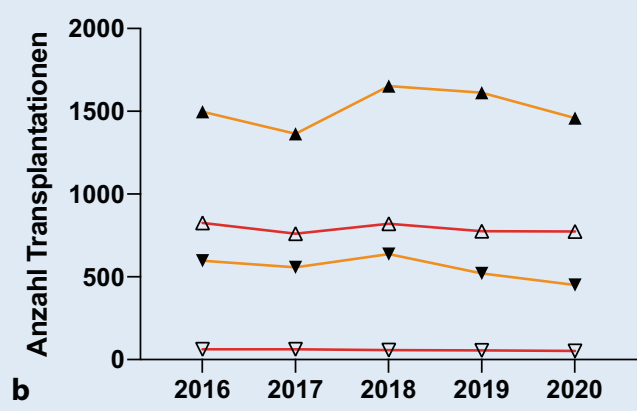

- Nierentransplantation, Lebendspende

- Nierentransplantation, Totenspende

$\nabla$ Lebertransplantation, Lebendspende

$\triangle$ Lebertransplantation, Totenspende

Transplantationen - Berlin

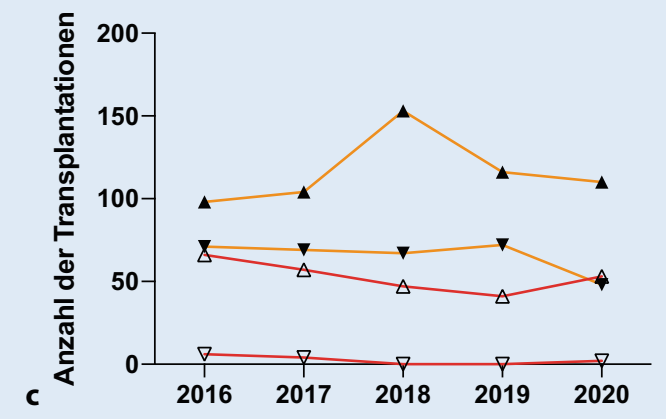

$\triangle$ Lebertransplantation, Totenspende

$\nabla$ Lebertransplantation, Lebendspende

^ Nierentransplantation, Totenspende

$\checkmark$ Nierentransplantation, Lebendspende

\section{Risikokonstellation perioperative SARS-CoV-2-Infektion}

SARS-CoV-2-Infektionen im perioperativen Setting von HPB-Eingriffen gehen mit einer erhöhten Rate an pulmonalen Komplikationen sowie einer erhöhten 30-TageMortalität einher, im Vergleich zur Kohorte von HPB-Patienten ohne SARS-CoV-2Exposition [2, 20]. Als weitere relevante Faktoren hierbei wurden das Alter über 70 Jahre, onkologische und Major-Eingriffe ermittelt [2]. Aus diesen Daten lässt sich eine ausgesprochene Fragilität der HPBchirurgischen Patienten ableiten [25]. Bis zu 7 Wochen nach einer Infektion mit SARSCoV-2 zeigen sich für postoperative Verläufe eine erhöhte Rate an pulmonalen Komplikationen sowie eine erhöhte Mortalität [14]. Dies gilt sowohl für Patienten, welche eine asymptomatische Infektion durchgemacht hatten, als auch für Patienten nach symptomatischer Infektion ohne residuelle Symptome. Eine entsprechende Auswertung spezifisch für HPB-Eingriffe steht nicht zur Verfügung. Eine Übertragbarkeit der Ergebnisse ist anzunehmen und die Anwendung durchaus relevant, da es sich meist um zeitkritische Indikationen handelt. Ein zeitweises Ausweichen auf interventionelle Therapieoptionen im Sinn eines Bridging-to-surgery-Konzeptes wird somit diskutiert. Eine abschließende wissenschaftliche Auswertung zu diesem Thema ist aktuell ausstehend und Umsetzungen müssen sicherlich der Situation vor Ort angepasst werden.

\section{Risikokonstellation Organ- transplantation}

In Analogie zu etablierten Daten, die eine Korrelation zwischen dem Vorhandensein einer Immunsuppression und einem erhöhten Risiko für schwere Verläufe infektiöser Erkrankungen beschreiben [18], wurde zunächst ein stark erhöhtes Risiko eines schweren COVID-19-Verlaufs für transplantierte Patienten postuliert. Erste Daten, publiziert im Juni 2020, berechneten für eine Kohorte transplantierter Patienten eine hohe COVID-19-bedingte 30-Tage-Mortalität von $28 \%$ (vgl. Mortalität der allgemeinen Bevölkerung ohne Vorerkrankung: < 4\% der < 60-Jährigen; 23-40\% der > 80-Jährigen; [1, 26]). Wei- 
tere Auswertungen zeigen jedoch ein differenzierteres Bild: Vor allem für Patienten nach Nieren- und Lungentransplantation bestätigte sich ein hohes Risiko für schwere COVID-19-Verläufe (Hazard Ratio von 4,6 und 3,5 für Krankenhausaufnahme und 7,1 und 6,2 für Mortalität [23\%]; [10]). Größere Kohortenstudien berichten von einer Mortalität für Nierentransplantierte von $20-32 \%[10,15,17]$.

\section{॥ Die hohe Sterblichkeit begründet sich durch die hohe Anzahl an Komorbiditäten}

Diese hohe Sterblichkeit begründet sich jedoch nicht nur durch die immunsuppressive Therapie oder die Transplantation an sich, sondern vielmehr durch die hohe Anzahl an Komorbiditäten [5]. Eine amerikanische Multicenterstudie beschreibt nach Propensity Score Matching eine Mortalität von $40 \%$ für Transplantierte (70\% Nierentransplantierte) vs. $43 \%$ für nichttransplantierte Patienten mit ähnlichem Profil an Vorerkrankungen [22]. Ein ähnliches Ergebnis zeigen Chaundry et al. für 47 Transplantierte, davon 39 Nierentransplantierte (Mortalität $40 \%$ vs. $48 \%$ für eine nichttransplantierte Vergleichsgruppe; [11]). Für Lebertransplantierte zeigt sich interessanterweise eine deutlich niedrigere Mortalität als für Nierentransplantierte. Die größte Auswertung hierzu umfasst 151 Lebertransplantierte aus 18 Ländern. Eine Mortalität von $19 \%$ für Lebertransplantierte wurde berechnet sowie eine entsprechende Mortalität für eine nichttransplantierte Kohorte nach Propensity Score Matching (u.a. Alter, Geschlecht, Übergewicht, Hypertonus; [7]).

Zusammenfassend muss der Einfluss einer Immunsuppression im Hinblick auf den Verlauf nach SARS-CoV-2-Infektion kontrovers diskutiert werden. Danzinger-Isakov et al. postulieren für die Frühphase einer Infektion eine Reduzierung der erworbenen Immunantwort und somit einen Nachteil in der Viruselimination [16]. Eine immunmodulatorische und antiinflammatorische Komponente könnte für eine spätere Phase sogar einen protektiven Effekt mit sich bringen [21]. Durch die Einführung von Dexamethason in die COVID19-Therapie konnte immerhin eine Senkung der Mortalität um $12 \%$ erreicht wer- den [30]. Eine schrittweise Reduktion der Immunsuppression gemäß der Schwere der Symptome ist häufig beschrieben, eine evidenzbasierte Strategie steht aktuell nicht zur Verfügung [21]. Für Transplantierte mit krankenhauspflichtigem COVID-19Verlauf wurde in bis zu $88 \%$ die Antimetabolitimmunsuppression pausiert, Kalzineurininhibitoren nur in $20-30 \%$ [10, 24].

\section{Ausblick: Impfung}

Einstimmig wird von Transplantationsorganisationen die priorisierte Impfung von Transplantationspatienten gefordert. Auch für Patienten mit HPB-Malignomen wird eine priorisierte Impfung gefordert [13]. In einer Modellrechnung ist die „number needed to vaccinate to prevent one COVID-19related death in 1 year" (NNV) für Tumorpatienten > 70 Jahre, welche eine Operation benötigten, am niedrigsten (NNV wahrscheinlichster Fall: 351; „best case“: 196, „worst case": 816) [12]. Genau in diesem Spektrum befindet sich der Großteil der Patienten im Bereich der HPB-Chirurgie.

\section{॥) Problematisch scheint die niedrige Serokonversion nach Organtransplantation zu sein}

Transplantationsgesellschaften favorisieren das Impfen von RNA-Impfstoffen für Immunsupprimierte, da eine höhere Wirksamkeit bei älteren Patienten gezeigt wurde. Sowohl Impfstoffe auf RNA-Basis als auch auf Basis von Vektorviren werden als sicher für transplantierte Patienten angesehen, auch wenn in den Zulassungsstudien der drei aktuell wichtigsten Impfstoffe - BNT162b2 mRNA vaccine (Pfizer/ BioNTech), mRNA-1273 (Moderna), ChAdOx1-nCoV-19 (AstraZeneca/University of Oxford) - immunsupprimierte Patienten ausgeschlossen wurden. Erste Daten für transplantierte Patienten beschreiben ein niedriges Nebenwirkungsprofil [8]. Problematisch scheint vielmehr die niedrige Serokonversion nach Organtransplantation zu sein. Für eine Kohorte von 436 Transplantationspatienten wurde eine Serokonversion von nur 17\% 20 Tage nach Impfung beschrieben [9]. Hohes Alter und Antimetabolitimmunsuppression waren hierbei mit einer noch niedrigeren Immunantwort assoziiert. Somit scheinen erweiterte Hygienebestimmungen für diese Patienten unabhängig vom Impfstatus indiziert.

\section{Fazit für die Praxis}

- Vor allem während der ersten pandemischen Welle - März bis Mai 2020 - kam es zu einem deutlichen Rückgang der Eingriffszahlen im Bereich der hepatopankreatobiliären (HPB-)Chirurgie und Organtransplantation. Der zu erwartende kompensatorische Anstieg in den Folgemonaten blieb meist aus.

- Es wurde eine Reduktion ambulanter Patientenvorstellungen und das Ausweichen auf alternative Therapieregime beschrieben. Der möglicherweise negative Einfluss dieser Effekte auf die Aufrechterhaltung der Versorgungsqualität bedarf einer stringenten Aufarbeitung.

- Sowohl Patienten nach Organtransplantation als auch Patienten der HPB-Chirurgie weisen aufgrund des Alters und Erkrankungsprofils ein erhöhtes Morbiditätsund Mortalitätsrisiko nach SARS-CoV2 (,severe acute respiratory syndrome coronavirus $\left.2^{\prime \prime}\right)$-Infektion auf.

- Die Rolle der Immunsuppression auf COVID-19(",coronavirus disease 2019“)assoziierte Morbidität und Mortalität ist nicht ausreichend verstanden. Priorisiertes Impfen beider Gruppen wird international gefordert, wenngleich die Serokonversion nach Transplantation niedrig ist.

- Erweiterte Hygienebestimmungen scheinen für diese Patienten unabhängig vom Impfstatus indiziert.

\section{Korrespondenzadresse}

Univ.-Prof. Dr. med. Johann Pratschke

Chirurgische Klinik, Campus Charité Mitte

| Campus Virchow-Klinikum, Charité -

Universitätsmedizin Berlin

Augustenburger Platz 1, 13353 Berlin,

Deutschland

johann.pratschke@charite.de

\section{Einhaltung ethischer Richtlinien}

Interessenkonflikt. K. Hillebrandt, N. Nevermann, B. Globke und S. Moosburner geben an, dass kein Interessenkonflikt besteht. M. Schmelzle erhielt Vortragshonorare von Merck Serono GmbH, Bayer AG, ERBE Elektromedizin GmbH und Amgen Inc.. M. S. erhielt Unterstützung für Operationskurse von ERBE Elektromedizin $\mathrm{GmbH}$, Johnson \& Johnson Medical $\mathrm{GmbH}$, Takeda Pharmaceutical Limited, Olympus K. K., Medtronic GmbH und Intuitive. J. Pratschke erhielt Honorare und Reisekostenerstattungen von Intuitive, Johnson \& Johnson Medical GmbH, Medtronic GmbH, Chiesi GmbH, La Fource Group, Merck und Neovii Pharmaceuticals. J. P. erhielt Honorare von Astellas, CHG 
Meridian, Falk Foundation, NOGGO, Pharma-ConsultPetersohn und Promedics. J. P. erhielt Reisekostenerstattungen von AFS Medical.

Für diesen Beitrag wurden von den Autoren keine Studien an Menschen oder Tieren durchgeführt. Für die aufgeführten Studien gelten die jeweils dort angegebenen ethischen Richtlinien.

\section{Literatur}

1. Akalin E, Azzi Y, Bartash R et al (2020) Covid19 and kidney transplantation. N Engl J Med 382:2475-2477. https://doi.org/10.1056/ nejmc 2011117

2. Almaadany FS, Samadov E, Namazov I et al (2020) Mortality and pulmonary complications in patients undergoing surgery with perioperative sars-cov-2 infection: an international cohort study. Lancet 396:27-38. https://doi.org/10.1016/ S0140-6736(20)31182-X

3. Alsaoudi T, Chung WY, Isherwood J et al (2020) HPB surgery in the time of COVID. Br J Surg 107:e588-e589. https://doi.org/10.1002/bjs. 12030

4. Amaddeo G, Brustia R, Allaire Met al (2021) Impact of COVID-19 on the management of hepatocellular carcinoma in a high-prevalence area. JHEP Rep 3:100199. https://doi.org/10.1016/j.jhepr.2020. 100199

5. Azzi Y, Bartash R, Scalea J et al (2021) COVID-19 and solid organ transplantation: a review article. Transplantation 105:37-55. https://doi.org/10. 1097/TP.0000000000003523

6. Bennett S, Søreide K, Gholami S et al (2020) Strategies for the delay of surgery in the management of resectable hepatobiliary malignancies during the COVID-19 pandemic. Curr Oncol 27:e501-e511. https://doi.org/10.3747/co.27.6785

7. Bhoori S, Rossi RE, Citterio D, Mazzaferro V (2020) COVID-19 in long-term liver transplant patients: preliminary experience from an Italian transplant centre in Lombardy. Lancet Gastroenterol Hepatol 5:532-533. https://doi.org/10.1016/S24681253(20)30116-3

8. Boyarsky BJ, Ou MT, Greenberg RS et al (2021) Safety of the first dose of SARS-coV-2 vaccination in solid organ transplant recipients. Transplantation. https://doi.org/10.1097/TP.0000000000003654

9. Boyarsky BJ, Werbel WA, Avery RK et al (2021) Immunogenicity of a single dose of SARS-coV-2 messenger RNA vaccine in solid organ transplant recipients. JAMA. https://doi.org/10.1001/jama. 2021.4385

10. Caillard S, Anglicheau D, Matignon M et al (2020) An initial report from the French SOT COVID registry suggests high mortality due to COVID19 in recipients of kidney transplants. Kidney Int 98:1549-1558. https://doi.org/10.1016/j.kint. 2020.08.005

11. Chaudhry ZS, Williams JD, Vahia A et al (2020) Clinical characteristics and outcomes of COVID-19 in solid organ transplant recipients: a cohort study. Am JTransplant 20:3051-3060. https://doi.org/10. 1111/ajt.16188

12. COVIDSurg Collaborative, GlobalSurg Collaborative (2021) SARS-CoV-2 vaccination modelling for safe surgery to save lives: data from an international prospective cohort study. Br J Surg. https://doi. org/10.1093/bjs/znab101

13. Cornberg M, Buti M, Eberhardt CS et al (2021) EASL position paper on the use of COVID-19 vaccines in

Impact of the COVID-19 pandemic on hepato-pancreato-biliary surgery and organ transplantation

Background and objective: Due to the coronavirus disease 2019 (COVID-19) pandemic extensive reorganization and limitation of resources within the healthcare system became inevitable. This review highlights the direct and indirect impact of the COVID19 pandemic on the fields of hepato-pancreato-biliary (HPB) surgery and organ transplantation incorporating the current literature and expert opinions published by national and international societies. Trends in surgical numbers were analyzed via the Eurotransplant Statistics Report Library and an in-center evaluation of HPB surgical procedures.

Results: For the fields of HPB surgery and organ transplantation a drastic decrease of procedures performed during the first wave of the pandemic was broadly reported. Interestingly, a compensatory increase of procedures was mostly not observed during the following months resulting in a slight overall decrease for 2020 compared to 2019 . Whether this trend was due to increased mortality because of postponed surgeries and altered treatment regimens cannot be ruled out at this time. A severe acute respiratory syndrome coronavirus 2 (SARS-CoV-2) infection is definitely associated with a complicated course, especially in the perioperative course and after transplantation. Discussion: Due to the increased risk profile of the named patient groups, there is an increased risk for a severe COVID-19 course. This must be considered when weighing up the treatment alternatives, protection recommendations and prioritization for vaccinations.

\section{Keywords}

SARS-CoV-2 · Perioperative infection · Care situation · Vaccination · Immunosuppression

patients with chronic liver diseases, hepatobiliary cancer and liver transplant recipients. J Hepatol. https://doi.org/10.1016/j.jhep.2021.01.032

14. COVIDSurg Collaborative, GlobalSurg Collaborative (2021) Timing of surgery following SARSCoV-2 infection: an international prospective cohort study. Anaesthesia. https://doi.org/10.1111/ anae. 15458

15. Cravedi P, Mothi SS, Azzi Y et al (2020) COVID19 and kidney transplantation: results from the TANGO international transplant consortium. Am J Transplant 20:3140-3148. https://doi.org/10. 1111/ajt.16185

16. Danziger-Isakov L, Blumberg EA, Manuel O, Sester M (2021) Impact of COVID-19 in solid organ transplant recipients. Am J Transplant 21:925-937. https://doi.org/10.1111/ajt.16449

17. Favà $A$, Cucchiari D, Montero N et al (2020) Clinical characteristics and risk factors for severe COVID19 in hospitalized kidney transplant recipients: a multicentric cohort study. Am J Transplant 20:3030-3041.https://doi.org/10.1111/ajt.16246

18. Fishman JA (2007) Infection in solid-organ transplantrecipients. NEnglJMed 357:2601-2614. https://doi.org/10.1056/nejmra064928

19. Loupy A, Aubert O, Reese PP et al (2020) Organ procurement and transplantation during the COVID-19 pandemic. Lancet 395:e95-e96. https:// doi.org/10.1016/S0140-6736(20)31040-0

20. Merath K, Hyer JM, Mehta R et al (2019) Use of perioperative epidural analgesia among medicare patients undergoing hepatic and pancreatic surgery. HPB 21:1064-1071. https://doi.org/10. 1016/j.hpb.2018.12.008

21. Merli $M$, Pasulo L, Perricone $G$ et al (2021) Impact of immunosuppressive therapy on the severity of COVID-19 in solid organ transplant recipients.
JInfect 82:414-451. https://doi.org/10.1016/j.jinf. 2020.10.024

22. Molnar MZ, Bhalla A, Azhar A et al (2020) Outcomes of critically ill solid organ transplant patients with COVID-19 in the United States. Am J Transplant 20:3061-3071.https://doi.org/10.1111/ajt.16280

23. Nevermann NFF, Hillebrandt KHH, Knitter S et al (2020) COVID-19 pandemic: implications on the surgical treatment of gastrointestinal and hepatopancreatobiliary tumours in Europe. $\mathrm{Br} J$ Surg 107:e301-e302. https://doi.org/10.1002/bjs. 11751

24. Pereira MR, Mohan S, Cohen DJ et al (2020) COVID19 in solid organ transplant recipients: initial report from the US epicenter. Am J Transplant 20:1800-1808. https://doi.org/10.1111/ajt.15941

25. Petrowsky H, Clavien PA (2005) Should we deny surgery for malignant hepato-pancreaticobiliary tumors to elderly patients? World J Surg 29:1093-1100. https://doi.org/10.1007/s00268005-1130-6

26. Reilev M, Kristensen KB, Pottegård A et al (2020) Characteristics and predictors of hospitalization and death in the first 11122 cases with a positive RTPCR test for SARS-coV-2 in Denmark: a nationwide cohort. Int J Epidemiol 49:1468-1481. https://doi. org/10.1093/ije/dyaa140

27. Ritschl PV, Nevermann N, Wiering L et al (2020) Solid organ transplantation programs facing lack of empiric evidence in the COVID-19 pandemic: a by-proxy society recommendation consensus approach. Am J Transplant 20:1826-1836. https:// doi.org/10.1111/ajt.15933

28. de Vries APJ, Alwayn IPJ, Hoek RAS et al (2020) Immediate impact of COVID-19 on transplant activity in the Netherlands. Transpl Immunol. https://doi.org/10.1016/j.trim.2020.101304 
29. Statistics library (2021) Eurotransplant. https:// www.eurotransplant.org/statistics/statistics-

library/.Zugegriffen: 18. Apr. 2021

30. RECOVERY Collaborative Group et al (2021)

Dexamethasone in hospitalized patients with

Covid-19. N Engl J Med 384:693-704. https://doi.

org/10.1056/nejmoa2021436

Hier steht eine Anzeige.

Springer 